\title{
CHARACTERIZATION OF HIPPOCAMPUS ON EPILEPTIC PATIENTS ON MRI USING TEXTURE ANALYSIS TECHNIQUES
}

\author{
Tasneem Abdulrazig Mohamed Sayed ${ }^{1}{ }^{\square}$ iD, Fatima Yousif Mohammed ${ }^{1}$ iD, \\ Dr. Maha Esmeal Ahmed ${ }^{1}$ iD \\ ${ }^{1}$ National University-Sudan, College of radiography and medical imaging sciences, Khartoum, \\ Sudan
}

DOI: https://doi.org/10.29121/granthaalayah.v9.i1.2021.2977

Article Type: Research Article

Article Citation: Tasneem Abdulrazig Mohamed Sayed, Fatima Yousif Mohammed, and Dr. Maha Esmeal Ahmed. (2021). CHARACTERIZATION OF HIPPOCAMPUS ON EPILEPTIC PATIENTS ON MRI USING TEXTURE ANALYSIS TECHNIQUES.

International Journal of Research GRANTHAALAYAH, 9(1), 164-168. https://doi.org/10.29121/granthaa layah.v9.i1.2021.2977

Received Date: 01 January 2021

Accepted Date: 31 January 2021

Keywords:

Hippocampus

Epilepsy

MRI

Texture Analysis

\section{ABSTRACT}

The aim of this study was to characterize the hippocampus in Sudanese epileptic patients in MR images using image texture analysis techniques in order to differentiate hippocampus between the normal and epileptic patient. There were two groups of the patients were examined by using Signal-GE 1.5Tesla MR Scanner which was used with patients with known epilepsy and normal T1 weighted brain. MRI finding patients, 101 and 105 patients respectively examined in period from December 2017March 2018, where the variables of the study were MRI images entered to the IDL program as input for further analysis, using window $3 * 3$ the images texture was extracted from hippocampus (head, body and tail) that include, mean, STD, variance, energy, and entropy then the comparison was made to differentiate between the normal and abnormal hippocampus. The extracted feature classified using linear discriminate analysis. The classification score function is used to classify the hippocampus classes was as flows:

Epileptic $=(.271 \times$ mean $)+(.026 \times$ variance $)+(7.475 \times$ Part $)-32.134$

Normal $=(.240 \times$ mean $)+(.052 \times$ variance $)+(2.960 \times$ Part $)-13.684$

The study confirmed that it's possible to differentiate between normal and epileptic hippocampus body, head, and tail in sagittal section texturally. The result showed that the classification result is best in the tail where higher classification accuracy will be achieved followed by body and then head.

\section{INTRODUCTION}

The hippocampus is an important structure situated in the temporal lobe and it is a part of the Limbic System. It has a strong role in the transition of memory from short term to long term memory [1]Damage to the hippocampus may result in loss of memory or epilepsy. E.g.: Hippocampus sclerosis (damage with scarring and atrophy of the hippocampus and surrounding cortex) is the main pathological substrate causing temporal lobe and the leading cause of localization related epilepsy.[2]Epilepsy is a chronic neurological condition characterized by recurrent epileptic seizures, there are two types of epilepsy: Generalized epilepsy and Focal epilepsy. [3] Epilepsy can be caused by neuronal migration abnormalities, infectious, genetic or immune causes. Magnetic resonance imaging (MRI) exams are a key element of the pre-surgical epilepsy work-up and identifications [4], MRI studies provide a

(C) 2021 The Author(s). This is an open access article distributed under the terms of the Creative Commons Attribution License, which permits unrestricted use, distribution, and reproduction in any medium, provided the original author and source are credited. 
tool for the study of structural changes over time to determine the effects of epilepsy on the brain [5]. Recently, it has been shown that the determination of structural and volumetric asymmetries in the human brain from MRI provides critical data for the diagnosis of focal abnormality. This has been the case with complex partial seizures attributable to hippocampus sclerosis and has been further applied to other brain regions for the same purpose. [6] Texture analysis is an important branch of digital image processing that has found application in several research areas, although a clear definition of texture does not exist, it can be understood as a group of image properties that relate to our intuitive notions of coarseness, rugosity, smoothness etc. [7] Texture analysis of MR images is a quantitative method that can be used to detect and quantify structural abnormalities in different tissues [8],[9]. It makes it possible to assess the degree of gray-tone modifications and the alterations of gray-tone spatial distribution in a given anatomic region of interest. This gray-tone variation is thought to correspond to underlying functional and anatomic changes, IDL (TEXTURE) [10]. This study was to characterize hippocampus on epileptic patients in the MR images using the texture analysis method.

\section{MATERIALS AND METHODS}

\section{Context}

Patient known pathological manifestation of epilepsy such as abnormal brain signal presented to radiology department of Al-Moalim Medical City for MRI scan, 101 and 105 patients respectively examined in period from December 2017 to March 2018.

The group of date selected from Signa-GE 1.5Tesla MR Scanner.T1-weighted images with $1 \mathrm{~mm}$ isotropic voxels were acquired for all subjects using a spoiled gradient echo sequence with flip angle $=35^{\circ}$, repetition time $(\mathrm{TR})=22 \mathrm{MS}$, echo time (TE) $=9 \mathrm{MS}$, matrix $=256 \times 220$, and field of view (FOV) $=23 \times 25 \mathrm{~cm}$.

Hippocampus is major part of the brain is elongated ridges on the floor of each ventricle. And has three part (head, body, tail). Hippocampus is play important role in limbic system is involved in the formation of emotion, memory, and the autonomic nerves system.

In the data collection and technique MR images were viewed by the Radiant, Ant. DICOM in computer to select the section of image and uploaded it into the computer-based software Interactive Data language (IDL) where the DICOM image converted to TIFF (JEPG) format and the user then clicks on areas represents the head, body and tail on hippocampus. In these areas a window of $3 \times 3$ pixel will be set and the first order statistics were calculated, which include mean, SD, energy and entropy. The algorithm scans the whole image using a window of $3 \times 3$ pixel and computes the first order statistics and computes the distance (the Euclidean distance) between the calculated features and the class's centers and assigns the window to the class with the lowest distance. Then the window interlaced one pixel and the same process stated over till the entire images were classified the data concerning the head, body and tail on hippocampus entered into SPSS to be statistically analyzed.

Texture analysis is technique used for the quantification of image texture it has been applied in MRI as computer aided diagnostic tool by repeating pattern of local variation in image intensity that mean characterization of regions in the image.

To generate a classification score data analysis using stepwise linear discriminate analysis select the most discriminate feature that can be used in the classification of normal and abnormal hippocampus and brain tissues. Then error bar plots using discriminate function was generated as well as classification accuracy and linear discriminate function equation to differentiate between normal and abnormal hippocampus and brain tissues for unseen images. 


\section{Results}

Abnormal

hippocampus
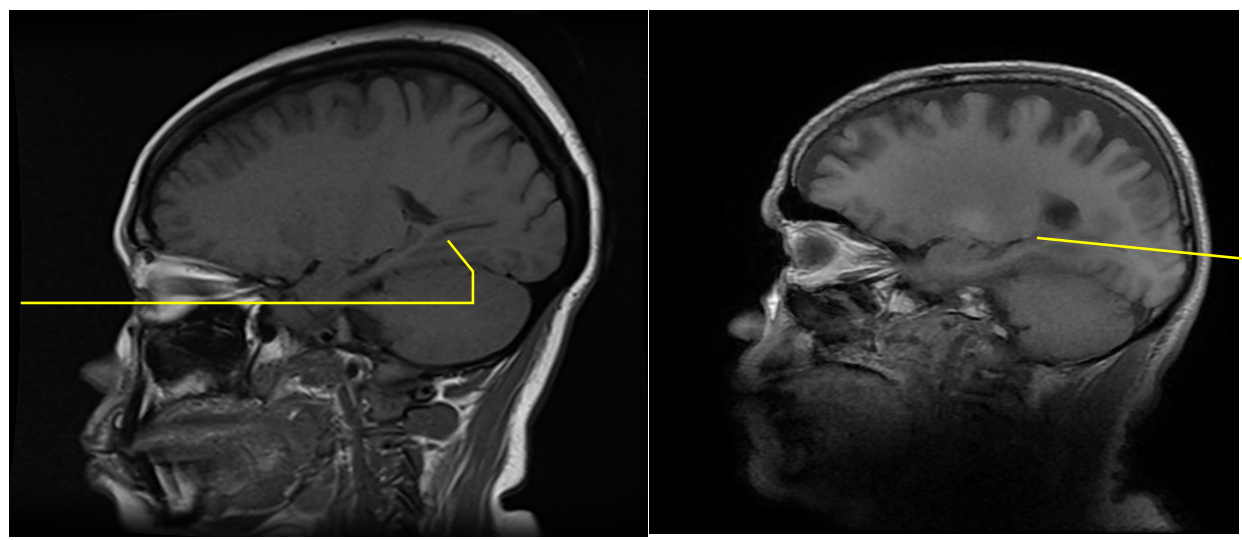

Normal

hippocamps

Image 1: Sagittal TI images for patient with epilepsy (left) and normal patient (right)

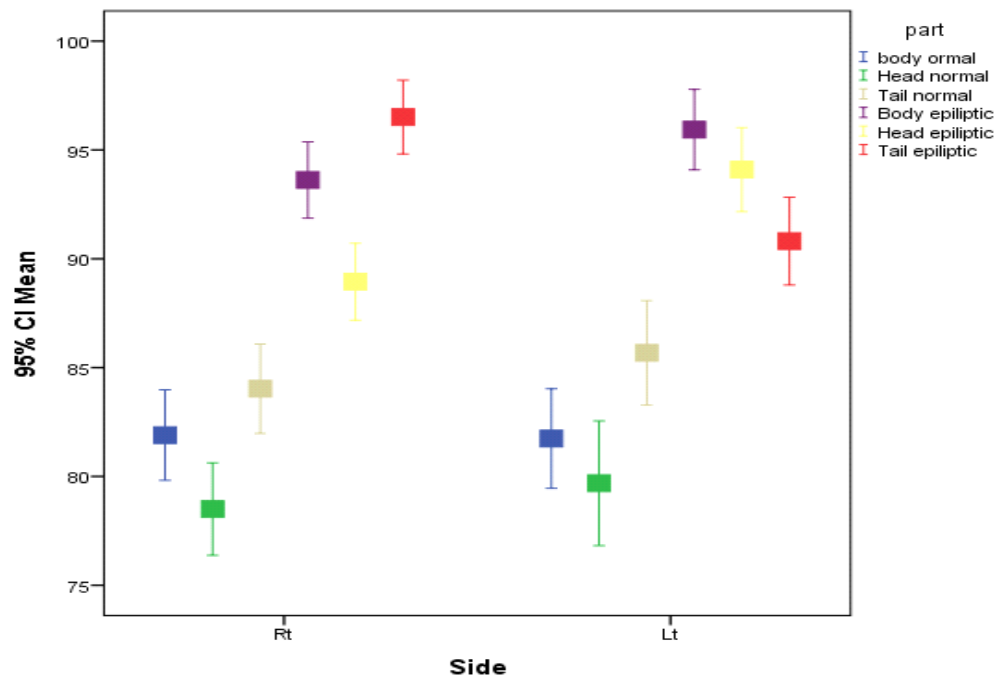

Figure 1: Error bar plot show the discriminate power of the mean textural feature distribution for the selected classes on T1 images for normal \& epileptic patients on hippocampus.

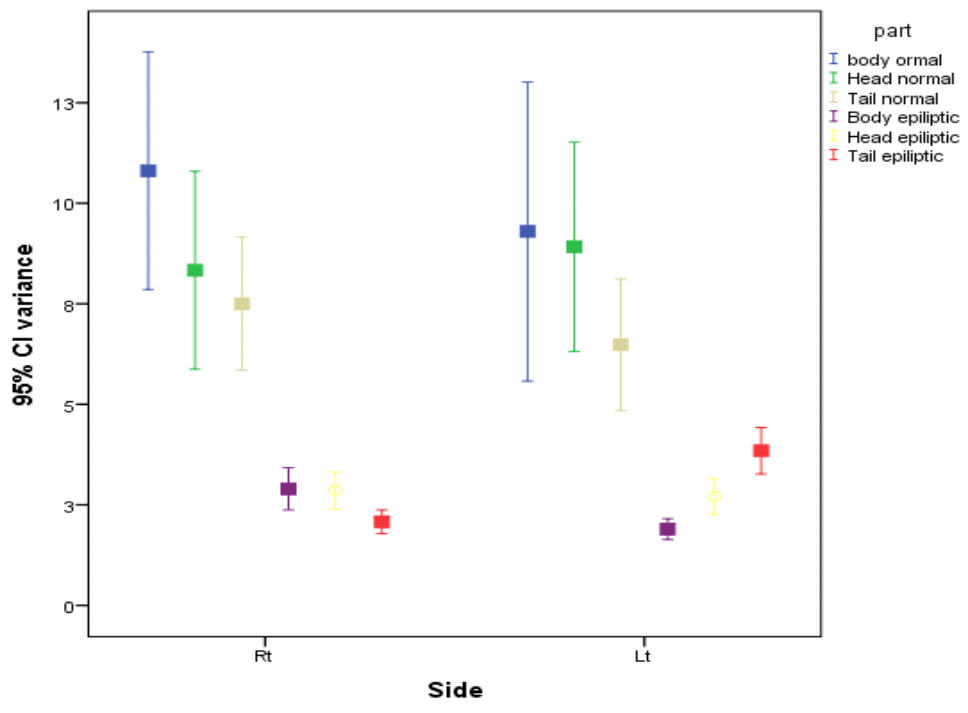

Figure 2: Error bar plot show the discriminate power of the variance textural feature distribution for the selected classes on T1 images for normal \& epileptic patients on hippocampus. 
Tasneem Abdulrazig Mohamed Sayed, Fatima Yousif Mohammed, and Dr. Maha Esmeal Ahmed

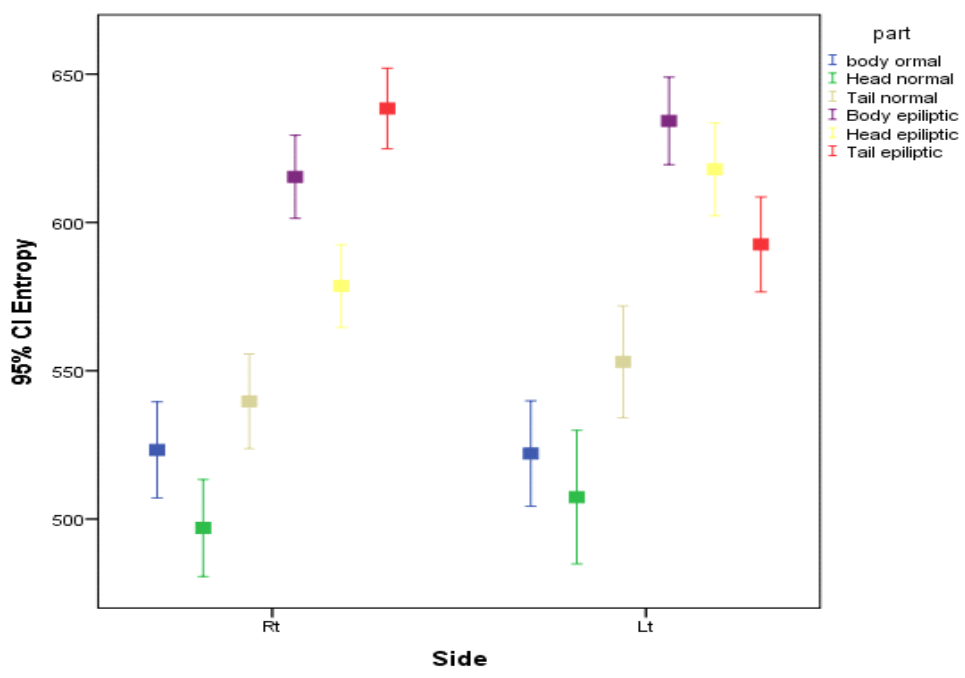

Figure 3: Error bar plot show the discriminate power of the Entropy textural feature distribution for the selected classes on T1 images for normal \& epileptic patients on hippocampus

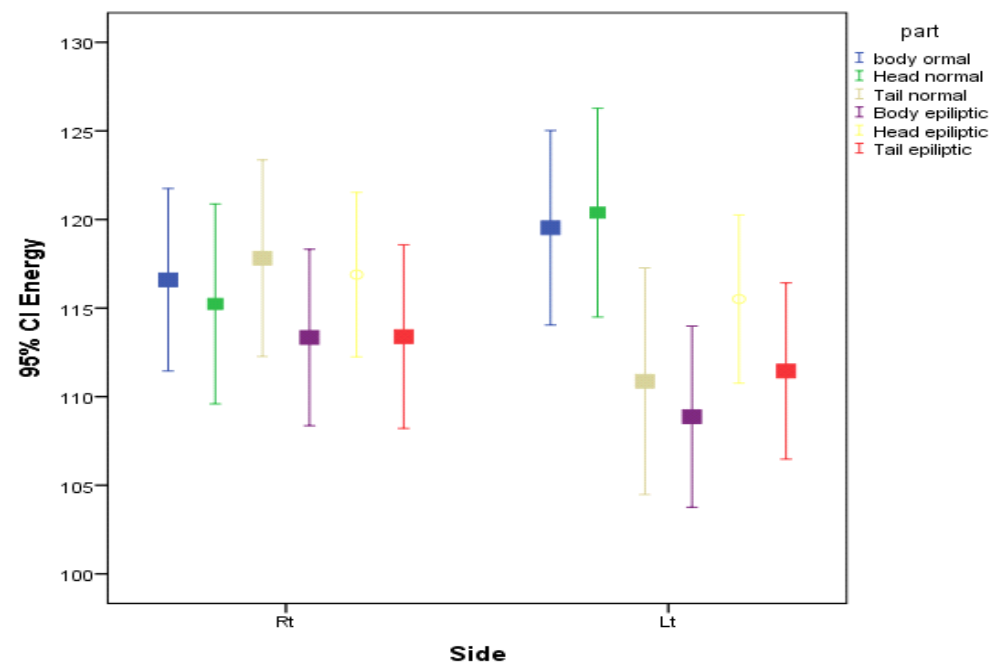

Figure 4: Error bar plot show the discriminate power of the energy textural feature distribution for the selected classes on T1 images for normal \& epileptic patients on hippocampus

\section{DISCUSSION}

The aim of this study is to characterize the hippocampus on epileptic patient in T1 magnetic resonance images by using texture analysis.

In this study there were three classes on hippocampus (head, body and tail) .On this classes we extract for features in each class (mean, entropy, energy and variance). To classify hippocampus in normal and abnormal (epileptic patient) using linear discriminate analysis. In fig (1) on T1 images the epileptic patient has the highest mean than the normal. In the RT side in epileptic patient the tail has the highest mean, but in LT side the body is the highest, while in normal the tail is highest in both (RT, LT)

In fig (2) on T1 images the epileptic patient has the less rang of scatter than normal. In the RT side in epileptic patient and normal is less range of scatter than the LT side. While LT side in normal the body has more rang of scatter but epileptic patient the tail has more rang of scatter than the RT side.

In fig (3) onT1 images the epileptic patient has the highest entropy than the normal. In the RT side in epileptic patient the tail has the highest entropy, but in LT side the body is the highest, while in normal the tail is highest in both (RT, LT). 
In fig (4) on T1 images the epileptic patient has the less energy than normal. In the RT side in epileptic patient and normal is less energy than the LT side. While LT side in normal the head has more energy but in epileptic patient the tail has more energy. The classification score function is used to classify the hippocampus classes was as flows:

Epileptic $=(.271 \times$ mean $)+(.026 \times$ variance $)+(7.475 \times$ Part $)-32.134$

Normal $=(.240 \times$ mean $)+(.052 \times$ variance $)+(2.960 \times$ Part $)-13.684$

This study confirmed that it's possible to differentiate between normal and epileptic hippocampus body, head, and tail in sagittal section texturally. The result showed that the classification result is best in the tail where higher classification accuracy will be achieved followed by body and then head. As to compare to Zuhal. (2017), study there is highly differentiate between normal and abnormal(epileptic) hippocampus by using texture analysis, and the tail of hippocampus is most accurate site in this differentiation

\section{CONCLUSION}

Epilepsy is common disease that affect the central nervous system, the range of causes of epilepsy are different at different ages and in different countries. Hippocampus sclerosis is one of causes of epilepsy. Texture analysis can provide useful information about the microstructure of the organ of interest. Finally using MR images and texture analysis can differentiate between hippocampus for patient with epilepsy from normal.

\section{SOURCES OF FUNDING}

This research received no specific grant from any funding agency in the public, commercial, or not-for-profit sectors.

\section{CONFLICT OF INTEREST}

The author have declared that no competing interests exist.

\section{ACKNOWLEDGMENT}

None.

\section{REFERENCES}

[1] Richard s.snell. Clinical neuroanatomay (2010)7th edition lippincott Williams \&willkins .pp. (300-305).

[2] David A. warrell (2003) Oxford textbook of medicine 4th edition.

[3] Yu, O., Roch, C., Namer, I. J., Chambron, J., \&Mauss, Y. (2002). Detection of late epilepsy by the texture analysis of MR brain images in the lithium-pilocarpine rat model. Magnetic resonance imaging (2010), 771-775.

[4] Rebecca Emily Feldman, Bradley Neil Delman,Puneet Singh Pawha, HadrienDyvorne,John Watson Rutland, JiyeounYoo, Madeline Cara Fields, Lara Vanessa Marcuse, PritiBalchandani

[5] T M Salmenpera, J S Duncan

[6] Jafari-Khouzani, K., Siadat, M. R., Soltanian-Zadeh, H., \&Elisevich, K. (2003, May). Texture analysis of hippocampus for epilepsy. In Proceedings of SPIE (Vol. 5031, pp. 279-288).

[7] R.M. Haralick Statistical and structural approaches to texture Proc IEEE, 67 (1979), pp. 788-804

[8] Lerski RA, Straughan K, Schad LR, et al. MR image texture analysis: an approach to tissue characterization. MagnReson Imaging 1993; 11: 873 - 87.

[9] Kjaer L, Ring P, Thomsen C, et al. Texture analysis in quantitative MR imaging: tissue characterisation of normal brain and intracranial tumours at 1.5 T. ActaRadiol 1995;36: $127-35$

[10] Bernasconi A, Antel SB, Collins DL, et al. Texture analysis and morphological processing of magnetic resonance imaging assist detection of focal cortical dysplasia in extra-temporal partial epilepsy. Ann Neurol 2001; 49: $770-5$. 